\title{
Phase measurement of quantum walks: application to structure theorem of the positive support of the Grover walk
}

\author{
Norio Konno \\ Department of Applied Mathematics, Faculty of Engineering \\ Yokohama National University \\ Hodogaya, Yokohama 240-8501, Japan \\ konno@ynu.ac.jp \\ Iwao Sato \\ Oyama National College of Technology \\ Oyama, Tochigi 323-0806, Japan \\ isato@oyama-ct.ac.jp \\ Etsuo Segawa
}

Graduate School of Environment and Information Sciences, Graduate School of Education Center Yokohama National University

Hodogaya, Yokohama 240-8501, Japan

segawa-etsuo-tb@ynu.ac.jp

Submitted: Jan 20, 2018; Accepted: Apr 30, 2019; Published: May 17, 2019

(C) The authors. Released under the CC BY-ND license (International 4.0).

\begin{abstract}
We obtain a structure theorem of the positive support of the $n$-th power of the time evolution of the Grover walk on $k$-regular graph whose girth is greater than $2(n-1)$. This structure theorem is provided by the parity of the amplitude of another quantum walk on the line which depends only on $k$. The phase pattern of this quantum walk has a curious regularity. We also exactly show how the spectrum of the $n$-th power of the time evolution of the Grover walk is obtained by lifting up that of the adjacency matrix to the complex plain.
\end{abstract}

Mathematics Subject Classifications: 05C50, 81Q99

\section{Introduction}

The Grover walk is one of the important quantum walk model for not only quantum search algorithm $[1,16,17]$ but also bridges connecting to the quantum graphs [27, 33], 
reversible random walks [13], and also graph zeta [20,25] and graph theory [23]. The time evolution of the Grover walk is explained by a discrete-time analogue of reflection and transmission of the wave at each junction, that is, the vertex [6]. To give more precise definition, we prepare notions of graphs. For given $G=(V, E)$, let $A=A(G)$ be the set of symmetric arcs induced by the edge set $E$. The element of $A$ is called an arc. The inverse arc of $a \in A$ is denoted by $\bar{a}$. The arc from a vertex $u$ to a vertex $v$ is denoted by $(u, v)$. For an arc $a=(u, v), o(a)=u$ and $t(a)=v$ are called the origin and the terminus of $a$, respectively. Furthermore, the edge joining $u$ and $v$ is denoted by $|a|=\{u, v\}$. Note that $|a|=|\bar{a}| \in E$. The degree of $u \in V$ is defined by $\operatorname{deg}(u)=|\{a \in A \mid t(a)=u\}|$. Remark that since we treat the symmetric digraph, that is, $a \in A$ iff $\bar{a} \in A$, then the degree can be expressed by $\operatorname{deg}(u)=|\{a \in A \mid o(a)=u\}|$. Of course, $\operatorname{deg}(u)$ is equal to the out degree of $u$.

The total Hilbert space $\mathcal{A}$ is generated by the symmetric arc set $A$ of the given graph $G$. The quantum coin assigned at each vertex $u$ produces the complex valued weight of the transmission and reflection rate so that this representation matrix is a $\operatorname{deg}(u)$ dimensional unitary operator. In particular, for the Grover walk case, the transmission rate is $2 / \operatorname{deg}(u)$, and the reflection rate is $2 / \operatorname{deg}(u)-1$. Then the Grover walk is defined as follows:

Definition 1 (Grover walk on $G=(\boldsymbol{V}, \boldsymbol{A})$ ).

1. The total Hilbert space: $\mathcal{A}:=\ell^{2}(A)=\{\psi: A \rightarrow \mathbb{C} \mid\|\psi\|<\infty\}$. Here the inner product is the standard inner product, that is, $\langle\psi, \varphi\rangle=\sum_{a \in A} \overline{\psi(a)} \varphi(a)$.

2. Time evolution $U: \mathcal{A} \rightarrow \mathcal{A}$ (unitary):

$$
(U \psi)(a)=\sum_{b: t(b)=o(a)}\left(\frac{2}{\operatorname{deg}(o(a))}-\delta_{b, \bar{a}}\right) \psi(b) .
$$

Let the the time evolution operator of the Grover walk and the $n$-th iteration of the Grover walk starting from the initial state $\psi_{0}$ be denoted by $U$ and $\psi_{n}$, respectively. We introduce two non-linear maps $\mu: \mathcal{A} \rightarrow \ell(A)$ and $\nu: \mathcal{A} \rightarrow[0,2 \pi]^{A}$ as follows: for $\psi \in \mathcal{A}$,

$$
\begin{aligned}
& (\mu(\psi))(a)=|\psi(a)|^{2}, \\
& (\nu(\psi))(a)=\arg (\psi(a)) .
\end{aligned}
$$

Due to the unitarity of the Grover walk, $\mu_{n}:=\mu\left(\psi_{n}\right)$ becomes a probability distribution when the norm of the initial state $\psi_{0}$ is unit. Main interest of the Grover walk has been the investigation of the sequence of $\mu_{n}$ 's: the typical behaviors of quantum walks drive from observing the behavior $\mu_{n}$, for example, the coexistence of linear spreading and localization e.g., [18, 32] and its stationary measure for infinite graphs e.g., [19], the efficiency to the quantum search algorithm e.g., [24] and its references therein and perfect state transfer e.g., $[7,26]$. However it seems that it is natural to investigate also the phase 
measurement $\nu_{n}$ 's. To explain it, let us prepare notion of the positive support of a real matrix $M$. For any $b \in A, \delta_{b} \in \mathcal{A}$ is the delta function such that

$$
\delta_{b}(a)= \begin{cases}1 & : a=b \\ 0 & : a \neq b\end{cases}
$$

The matrix element $(M)_{b, a}$ is denoted by $\left\langle\delta_{b}, M \delta_{a}\right\rangle=\left(M \delta_{a}\right)(b)$. Let the positive support of a real matrix $M ; S(M)$, be defined by

$$
(S(M))_{b, a}= \begin{cases}1 & :(M)_{b, a}>0 \\ 0 & :(M)_{b, a} \leqslant 0\end{cases}
$$

Indeed, we focus on this $\nu$ in this paper since the map $\nu$ plays a key role to give the structure theorem of the positive support of the Grover walk. Taking the positive support of the Grover walk is first motivated by the fact of a direct connection between the Ihara zeta function and the positive support of the time evolution of the Grover walk [25]:

$$
\zeta_{G}(u)=\operatorname{det}\left(1-u S\left(U_{G}\right)\right)^{-1}
$$

where $\zeta_{G}(u)$ is the Ihara zeta function and $U_{G}$ is the time evolution of the Grover walk induced by graph $G$. Zeta function of a graph was started from Ihara zeta function of a graph [15]. Originally, Ihara defined $p$-adic Selberg zeta functions of discrete groups, and showed that its reciprocal is a explicit polynomial. Serre [28] pointed out that the Ihara zeta function is the zeta function of the quotient $T / \Gamma$ (a finite regular graph) of the one-dimensional Bruhat-Tits building $T$ (an infinite regular tree) associated with $G L\left(2, k_{p}\right)$. A zeta function of a regular graph $G$ associated with a unitary representation of the fundamental group of $G$ was developed by Sunada [30, 31]. Hashimoto [11] treated multivariable zeta functions of bipartite graphs. Bass [2] generalized Ihara's result on the Ihara zeta function of a regular graph to an irregular graph, and showed that its reciprocal is again a polynomial. New proofs for Bass' formula were given in [5, 21, 29]. Furthermore, Konno and Sato [20] presented an explicit formula for the characteristic polynomial of the time evolution of the Grover walk on a graph $G$ by using the determinant expression for the second weighted zeta function of $G$, and directly obtained spectra for the time evolution of the Grover walk on $G$.

The second motivation to take the positive support of the time evolution of the Grover walk is that the spectrum of the positive support of the time evolution of the Grover walk has been believed to be a strong tool for the graph isomorphism problem:

Conjecture 2. [4] Let $G$ and $H$ be strongly regular graphs. Then

$$
G \cong H \Leftrightarrow \sigma\left(S\left(U_{G}^{3}\right)\right)=\sigma\left(S\left(U_{H}^{3}\right)\right),
$$

where $\sigma(A)$ is the spectrum of $A$. 
Recently, a counter example of the graph having a large number of vertices are suggested by a combination of theoretical and numerical method [9]. However finding the class of strongly regular graphs which conserves the conjecture is still an interesting open problem.

Therefore taking together with the above two motivations of the positive support of the Grover walk, we can naturally extend the Ihara zeta function as follows:

$$
\zeta_{G}^{(n)}(u)=\operatorname{det}\left(1-u S\left(U^{n}\right)\right)^{-1}
$$

There are structure theorems for $S\left(U^{2}\right)$ and $S\left(U^{3}\right)$ as follows:

Theorem 3. Structure theorem for $S\left(U^{2}\right)$ and $S\left(U^{3}\right)$

1. ([8]) If $G$ is a graph without leaves, then

$$
S\left(U^{2}\right)=I+S(U)^{2}
$$

2. ([12]) additionally, if the girth is greater than $4 ; g(G)>4$, and it is $k$-regular, then

$$
S\left(U^{3}\right)=S(U)^{3}+{ }^{\top} S(U)
$$

Here the girth $G ; g(G)$, is the smallest length of cycle of $G$.

Moreover a beautiful structure theorem of $S\left(U^{3}\right)$ in [10] for the strongly regular graph is obtained. In this paper, we consider the structure theorem of $S\left(U^{n}\right)$ for general $n$. The graph in our setting should have a large girth $g(G)>2(n-1)$ with the degree's regularity which includes the setting of [12].

In our main theorem, $S\left(U^{n}\right)$ is expressed by a linear combination of $S(U)^{k}, J S(U)^{k}$, ${ }^{\top} S(U)^{k}$ and $J^{\top} S(U)^{k}(k=0, \ldots, n)$, where $J: \ell^{2}(A) \rightarrow \ell^{2}(A)$ such that $(J \psi)(a)=\psi(\bar{a})$, ${ }^{\top} M$ is the transpose of $M$. The advantage point of this expression is that we express the spectrum of $S\left(U^{n}\right)$ by using the spectrum of the adjacency matrix of $G$. Thus we can see how the spectrum of $S\left(U^{n}\right)$ is lifted up to the complex plane from the spectrum of the adjacency matrix on the real line. See Theorem 13 and Fig. 7, we obtain the support of the non-trivial zero's of $1 /\left(z^{|A|} \zeta_{G}^{(n)}\left(z^{-1}\right)\right)$. On the other hand, the negative point is that $\sigma\left(S\left(U^{n}\right)\right)$ with $g(G)>2(n-1)$ cannot determine the graph isomorphism since there are graphs which are not isomorphism but cospectral of the positive support due to this "advantage" point. As is suggested by the appearance of the Hadamard product in [10], only the spectrum of the adjacency matrix cannot determine $S\left(U^{n}\right)$ in general. However we believe that our main theorem brings a new study motivation of quantum walks investigating the phase observation $\nu$; note that the operation taking support is converted to the phase observation problem, that is, letting $\psi_{n}^{(b)}(a)$ be the $n$-th iteration of the Grover walk at a starting from $b$, that is, $\psi_{n}^{(b)}(a)=\left(U^{n} \delta_{b}\right)(a)$, then we have $\left(S\left(U^{n}\right)\right)_{a, b}=1 \Leftrightarrow \nu\left(\psi_{n}^{(b)}\right)(a)=0$.

We show that in our setting, this phase observation problem can be switched to solving the phase pattern $\left\{\nu_{n}\right\}_{n \in \mathbb{N}}$ of the discriminant quantum walk on the one-dimensional lattice defined below, which is another quantum walk model. As we will see, this phase 
pattern informs us an exact expression for the structure theorem and seems to have a curious regularity (see Figs. 2-3). However a complete decode of this pattern still remains as one of the interesting open problems induced by the operation of the positive support. The following is the definition of the discriminant quantum walk:

Definition 4. Discriminant quantum walk. Let $k \geqslant 2$ be a natural number.

1. Hilbert space: $\ell^{2}\left(\mathbb{Z} ; \mathbb{R}^{2}\right)=\left\{\psi: \mathbb{Z} \rightarrow \mathbb{R}^{2} \mid \sum_{x \in \mathbb{Z}}\|\psi(x)\|^{2}<\infty\right\}$.

2. Time evolution: Let $|L\rangle:={ }^{\top}[1,0]$ and $|R\rangle:={ }^{\top}[0,1]$ and $\langle L|=| L\rangle^{*},\langle R|=| R\rangle^{*}$. For $x \in \mathbb{Z}$, define $H_{m}(x)$ based on the sign of $x$ :

$$
H_{m}(x)=\left\{\begin{array}{cc}
{\left[\begin{array}{cc}
2 \sqrt{k-1} / k & -1+2 / k \\
1-2 / k & 2 \sqrt{k-1} / k
\end{array}\right]: x \geqslant 0,} & \\
{\left[\begin{array}{cc}
2 \sqrt{k-1} / k & 1-2 / k \\
-1+2 / k & 2 \sqrt{k-1} / k
\end{array}\right]} & : x<0 .
\end{array}\right.
$$

Let $P(x)=|L\rangle\left\langle L\left|H_{m}(x), Q(x)=\right| R\right\rangle\langle R| H_{m}(x)$ given initial state $\phi_{0}(x)=\delta_{0}(x)|R\rangle$. The state after $n$-step is $\phi_{n}=W \phi_{n-1}$, where

$$
(W \phi)(x)=P(x+1) \phi(x+1)+Q(x-1) \phi(x-1)
$$

for any $\phi \in \ell^{2}(A)$.

3. Phase measure: Letting $\phi \in \ell^{2}\left(\mathbb{Z} ; \mathbb{R}^{2}\right)$, we put $\phi(x):={ }^{\top}[\phi(x ; L), \phi(x ; R)] \in \mathbb{R}^{2}$. Then the phase measure $\nu: \ell^{2}\left(\mathbb{Z} ; \mathbb{R}^{2}\right) \rightarrow\{0, \pi, \varnothing\}^{\mathbb{Z} \times\{L, R\}}$ is the operation to pick up the argument of $\phi \in \ell^{2}\left(\mathbb{Z} ; \mathbb{R}^{2}\right)$, that is,

$$
(\nu(\phi))(x ; N)=\left\{\begin{array}{ll}
0 & : \phi(x ; N)>0 \\
\varnothing & : \phi(x ; N)=0, \\
\pi & : \phi(x ; N)<0
\end{array} \quad(N \in\{L, R\})\right.
$$

Here we regard $(x ; L)$ and $(x ; R)(x \in \mathbb{N})$ as $\operatorname{arcs}$ of $\mathbb{Z}$ as follows: $(x ; L)=(x+1, x)$ and $(j, R)=(x-1, x)$.

Now we are ready to give our main theorem as follows:

Theorem 5. Let $\phi_{n} \in \ell^{2}\left(\mathbb{Z} ; \mathbb{C}^{2}\right)$ and $\nu: \ell^{2}\left(\mathbb{Z} ; \mathbb{C}^{2}\right) \rightarrow\{0, \pi, \varnothing\}^{\mathbb{Z} \times\{L, R\}}$ be the above. Let $\epsilon_{j}, \tau_{j} \in\{0,1\}(j \in \mathbb{Z})$ be the indicator functions with respect to the signs of $\phi_{n}(j ; R)$ and $\phi_{n}(j ; L)$, respectively such that

$$
\epsilon_{j}=\left\{\begin{array}{ll}
1 & :\left(\nu\left(\phi_{n}\right)\right)(j ; R)=0 \\
0 & : \text { otherwise }
\end{array}, \tau_{j}= \begin{cases}1 & :\left(\nu\left(\phi_{n}\right)\right)(j-1 ; L)=0 \\
0 \quad: \text { otherwise }\end{cases}\right.
$$


Moreover let $J$ be defined by $(J \psi)(a)=\psi(\bar{a})$ for any $\psi \in \ell^{2}(A)$ and $a \in A$. Under the assumption $g(G)>2(n-1)$, and the regularity $k$, we have

$$
S\left(U^{n}\right)=\sum_{j=0}^{n}\left(\epsilon_{j} S(U)^{j}+\tau_{j} J S(U)^{j}\right)+\sum_{j=1}^{n-1}\left(\epsilon_{-j}^{\top} S(U)^{j}+\tau_{-j} J^{\top}\left(S(U)^{j}\right)\right) .
$$

This paper is organised as follows. We provide the phase pattern of the discriminant quantum walk in section 2, which is useful to obtain the exact form of RHS of Theorem 5 for each $n$. We put $\nu_{n}:=\nu\left(\phi_{n}\right)$. The sequence of $\left\{\nu_{n}\right\}_{n}$ seems to depict a kind of interesting regular pattern when we see it up to large time step. Section 3 is devoted to show the proof of our main theorem. Finally we give the spectral orbit of $S\left(U^{n}\right)$ with respect to the adjacency matrix of $G$ for $g(G)>2(n-1)$ and the degree $k$.

\section{Demonstration of the phase pattern}

In this section, we provide the phase pattern of the discriminant quantum walk. By Theorem 5, it is convenient to give the following one-to-one correspondence between $\{(j ; R),(j ; L) \mid j \in \mathbb{Z}\}$ and $\left\{S(U)^{j},{ }^{\top}\left(S(U)^{j}\right), J S(U)^{j}, J^{\top}\left(S(U)^{j}\right) \mid j \in \mathbb{Z}_{\geqslant 0}\right\}$

$$
\begin{aligned}
& (j ; R) \leftrightarrow \begin{cases}S(U)^{j} & : j \geqslant 0 \\
\top\left(S(U)^{|j|}\right) & : j<0\end{cases} \\
& (j ; L) \leftrightarrow \begin{cases}J S(U)^{j+1} & : j \geqslant 0 \\
J^{\top}\left(S(U)^{|j+1|}\right) & : j<0\end{cases}
\end{aligned}
$$

We put the matrix valued weights associated with left and right movings by

$$
\begin{aligned}
& P(x)= \begin{cases}P_{+}=\left[\begin{array}{cc}
2 \sqrt{k-1} / k & -1+2 / k \\
0 & 0
\end{array}\right] & : x \geqslant 0, \\
P_{-}=\left[\begin{array}{cc}
2 \sqrt{k-1} / k & 1-2 / k \\
0 & 0
\end{array}\right] & : x<0,\end{cases} \\
& Q(x)= \begin{cases}Q_{+}=\left[\begin{array}{cc}
0 & 0 \\
1-2 / k & 2 \sqrt{k-1} / k
\end{array}\right] & : x \geqslant 0, \\
Q_{-}=\left[\begin{array}{cc}
0 & 0 \\
-1+2 / k & 2 \sqrt{k-1} / k
\end{array}\right] & : x<0,\end{cases}
\end{aligned}
$$

For $n=1$, computing $\phi_{1}=W \phi_{0}$, we have $\phi_{1}(1)=Q_{+}|R\rangle$ and $\phi_{1}(-1)=P_{+}|R\rangle$, that is,

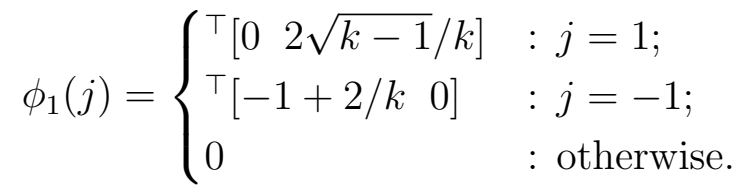


Thus the phase measurement results are

$$
\left(\nu\left(\phi_{1}\right)\right)(j ; R)=\left\{\begin{array}{ll}
0 & : j=1 \\
\varnothing & : \text { otherwise }
\end{array}, \quad\left(\nu\left(\phi_{1}\right)\right)(j ; L)= \begin{cases}\pi & : j=-1 \\
\varnothing & : \text { otherwise }\end{cases}\right.
$$

Since $(1 ; R)$ is the unique arc such that the phase value is 0 , that is, $\epsilon_{j}=1(j=1), \epsilon_{j}=0$ $(j \neq 1)$, and $\tau_{j}=0$ for any $j \in \mathbb{Z}$, then inserting them into (5), we check the trivial equation $S(U)=S(U)$. We define $\nu_{n}: A \rightarrow\{\boldsymbol{\square}, \square\}$ by

$$
\nu_{n}(a)= \begin{cases}\mathbf{\square} & :\left(\nu\left(\phi_{n}\right)\right)(a)=0 \\ \square & : \text { otherwise }\end{cases}
$$

Let us consider $n=2$ case. To obtain RHS of (5) for $n=2$ case explicitly, we check the arcs colored by "ם". Since $\phi_{2}(-2)=P_{-} P_{+}|R\rangle, \phi_{2}(2)=Q_{+}^{2}|R\rangle$ and $\phi_{2}(0)=\left(Q_{-} P_{+}+\right.$ $\left.P_{+} Q_{+}\right)|R\rangle$, examining the phases, we have

$$
\left(\nu\left(\phi_{2}\right)\right)(2 ; R)=0,\left(\nu\left(\phi_{2}\right)\right)(0 ; L)=\pi,\left(\nu\left(\phi_{2}\right)\right)(0 ; R)=0,\left(\nu\left(\phi_{2}\right)\right)(-2 ; L)=\pi
$$

which implies all the arcs colored by "ם" at time $n=2$ are $(2 ; R)$ and $(0 ; R)$. Then using the relation (6), we have

$$
S\left(U^{2}\right)=I+S(U)^{2}
$$

Now we understand that we only need to focus on the sequence of $\left\{\nu_{n}\right\}_{n \in \mathbb{N}}$ to obtain the explicit expression for RHS of (5) at each time. Figure 1 depicts the $\nu_{n}$ 's up to $n=4$, that is, $\left\{\nu_{1}, \nu_{2}, \nu_{3}, \nu_{4}\right\}$. Each cell corresponds to $(n,(j ; N))$ with $n \in \mathbb{N}, j \in \mathbb{Z}$ and $N \in\{L, R\}$. If $\nu_{n}(j ; N)=\mathbf{\square}$, that is, $\epsilon_{j}=1$ for $N=R$ and $\tau_{j}=1$ for $N=L$, then the corresponding cell color is black, otherwise the color is white. Referring the pattern of Fig. 1 and using (6), we can easily obtain the structure theorem for $n=3$ and $n=4$ :

$$
\begin{aligned}
& S\left(U^{3}\right)={ }^{\top} S(U)+S(U)^{3}, \\
& S\left(U^{4}\right)={ }^{\top} S(U)^{2}+I+S(U)^{4} .
\end{aligned}
$$

After $n=5$, a condition analysis arises with respect to the degree $k$ for example,

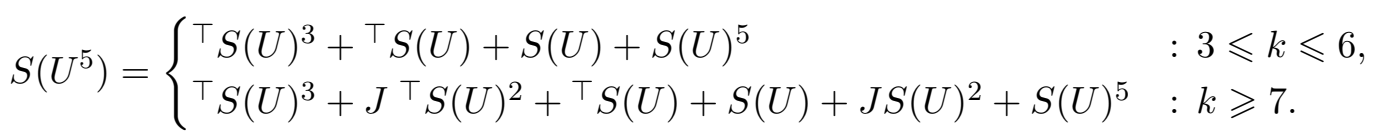

$$
\begin{aligned}
& S\left(U^{6}\right)= \begin{cases}\top S(U)^{4}+{ }^{\top} S(U)^{2}+I+S(U)^{2}+S(U)^{6} & : k=3,4, \\
{ }^{\top} S(U)^{4}+J^{\top} S(U)^{3}+{ }^{\top} S(U)^{2}+I+S(U)^{2}+J S(U)^{3}+S(U)^{6} & : 5 \leqslant k \leqslant 11 \\
{ }^{\top} S(U)^{4}+J^{\top} S(U)^{3}+I+S(U)^{2}+J S(U)^{3}+S(U)^{6} & : 12 \geqslant k .\end{cases}
\end{aligned}
$$

Figures 2(a)-2(c) are the phase patterns of $\nu_{n}$ 's for $k=20$ up to $n=10,20$ and 100, respectively. According to the result on the phase pattern, we can divide $\Xi:=\{(n, x) \in$ $\mathbb{N} \times \mathbb{Z}|| x \mid \leqslant 2 n\}$ plane into three regions $(A),(B)$ and $(C)$ : there exists $0<c<1$ such that 
1. Region $(A)$ : around the origin;

2. Region $(B):\{(n, x) \in \Xi|0<| x \mid / n \leqslant 2 c\}$;

3. Region $(C):\{(n, x) \in \Xi|2 c<| x \mid / n \leqslant 2\}$.

In Region $(A)$, we can observe a kind of check pattern, and in Region $(C)$, there is some regularity while complex pattern appears in Region $(B)$. The value $c$ seems to be $2 \sqrt{k-1} / k$ which is the diagonal part of the discriminant quantum walk's coin. See Figures 3(a)-3(c) for $k=20, k=10$ and $k=3$ cases until $n=500$. We can show the check pattern of Region $(A)$ in the forth coming paper [3] using the fact that the localization factor of the Grover walk is the infinite energy flow of the given graph [14]. We believe that a mathematical formulation of this phase pattern and also rigorous proof of each pattern are candidate of future's interesting problems.

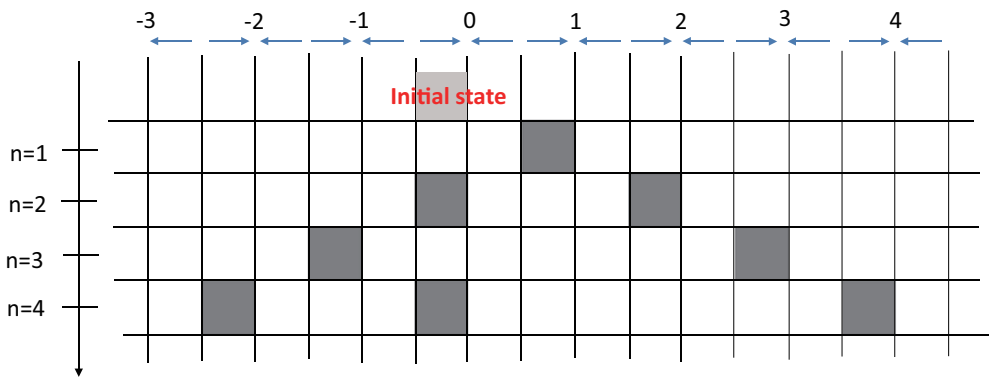

Figure 1: The phase pattern up to $n=4$ for $k=20$ : Each cell corresponds to $(n,(j ; N))$ with $n \in \mathbb{N}, j \in \mathbb{Z}$ and $N \in\{L, R\}$. If $\nu_{n}(j ; N)=\mathbf{\square}$, that is, $\epsilon_{j}=1$ for $N=R$ and $\tau_{j}=1$ for $N=L$, then the corresponding cell color is black, otherwise the color is white.

\section{Proof of main theorem}

First, we prepare notations for the proof of the theorem. Secondly, we give the proof for $k$-regular tree case. After this consideration, we address to the proof for $k$-regular graph with $g(G)>2(n-1)$ by using the local tree structure. See Fig 6 for the fundamental idea of the proof.

\subsection{Preliminary for the proof of main theorem}

For given $G=(V, E)$, let $A=A(G)$ be the set of symmetric arcs induced by the edge set $E$. A sequence of $\operatorname{arcs}\left(a_{1}, \ldots, a_{r}\right)$ in $G$ satisfying $t\left(a_{1}\right)=o\left(a_{2}\right), \ldots, t\left(a_{r-1}\right)=o\left(a_{r}\right)$ is called an $r$-length walk or simply a walk. The length of a shortest path from $u$ to $v$ is denoted by $\operatorname{dist}(u, v)$. If $t\left(a_{r}\right)=o\left(a_{1}\right)$, then this is called a $r$-length closed path. A back track of a closed path $\left(b_{0}, \ldots, b_{s-1}\right)$ is a subsequence $\left(b_{j}, b_{j+1}\right)$ with $b_{j}=\bar{b}_{j+1}$, where $j \in \mathbb{Z} / s \mathbb{Z}$. If there are no back tracks in a closed path, the closed path is called a cycle. 


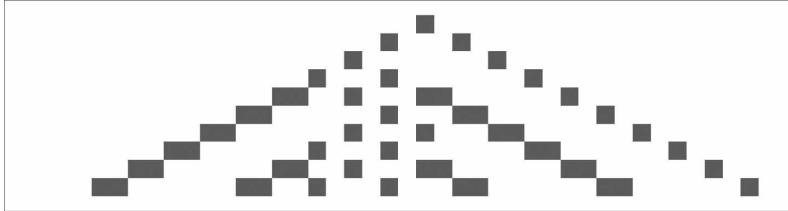

(a)The phase pattern up to $n=10$

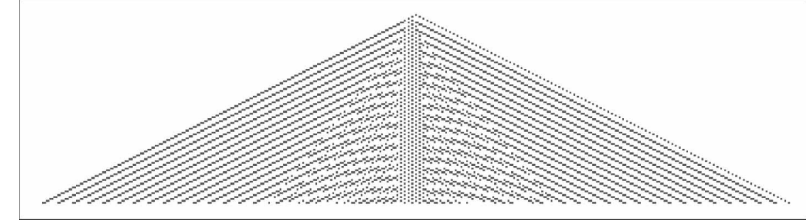

(c)The phase pattern up to $n=100$

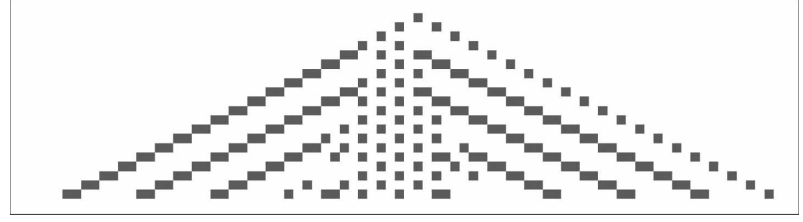

(b)The phase pattern up to $n=20$

Figure 2: Figures (a),(b) and (c) are the phase patterns up to $n=10, n=20$ and $n=100$, respectively for $k=20$ case

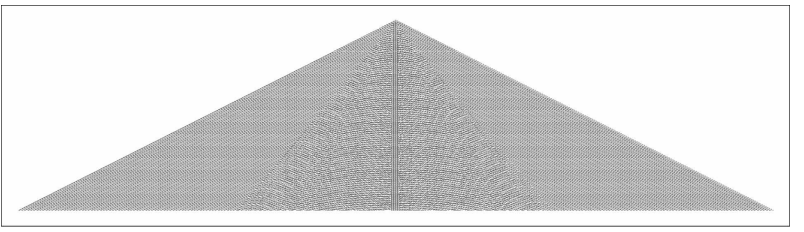

The phase pattern for $k=20$

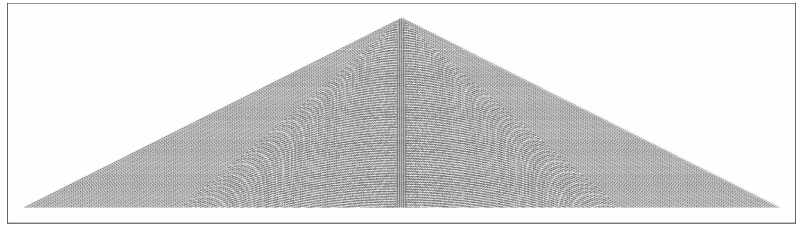

The phase pattern for $k=10$

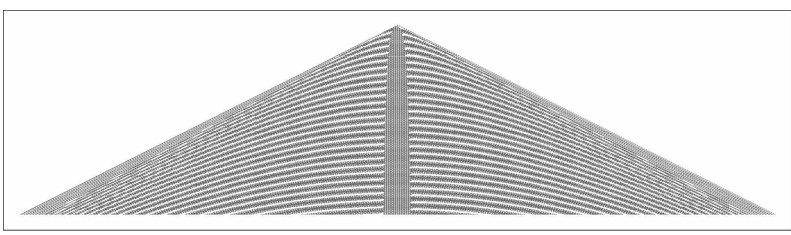

The phase pattern for $k=3$

Figure 3: Figures (a),(b) and (c) are the phase patterns Upton $n=500$, and whose degrees are $k=3, k=10$ and $k=20$, respectively. The value $c$ which determines the boundary of the region $B$ and $C$ increases when $k$ approaches to a small value. The value $c$ seems to be $2 \sqrt{k-1} / k$ which is the diagonal part of the discriminant quantum walk's coin. 
If $G$ has no cycles and $G$ is connected, then $G$ is called a tree. When $G$ is a tree, then the depth of the tree is the minimum height, where the height of the tree with a root $o \in V$ is defined by the largest distance from $o$. The $m$-level set of the tree with the root $o$ is the set of all the vertices whose distance from $o$ is $m$. The girth denoted by $g(G)$ is the length of a shortest cycle in $G$; when $G$ is a tree, we define $g(G)=\infty$.

We define a distance of pair of $\operatorname{arcs}(a, b)$ as follows:

$$
\operatorname{dist}(a, b):=\min \{\operatorname{dist}(t(a), o(b)), \operatorname{dist}(t(a), t(b)), \operatorname{dist}(o(a), t(b)), \operatorname{dist}(o(a), o(b))\} .
$$

Every pair of arcs $(a, b)$ has at least one of the following positional relations (see also Fig. 4):

Let $u \stackrel{j}{\sim} v$ denote $\operatorname{dist}(u, v)=j$ for $u, v \in V$. Then for $|a| \neq|b|$,

1. ${ }^{\exists} j_{1} \in \mathbb{N} \cup\{0\}$ such that $\operatorname{dist}(a, b)=j_{1}$ with $t(a) \stackrel{j_{1}}{\sim} o(b)$;

2. ${ }^{\exists} j_{2} \in \mathbb{N} \cup\{0\}$ such that $\operatorname{dist}(a, b)=j_{2}$ with $t(a) \stackrel{j_{2}}{\sim} t(b)$;

3. ${ }^{\exists} j_{3} \in \mathbb{N} \cup\{0\}$ such that $\operatorname{dist}(a, b)=j_{3}$ with $o(a) \stackrel{j_{3}}{\sim} t(b)$;

4. ${ }^{\exists} j_{4} \in \mathbb{N} \cup\{0\}$ such that $\operatorname{dist}(a, b)=j_{4}$ with $o(a) \stackrel{j_{4}}{\sim} o(b)$;

and for $|a|=|b|$, (5) $a=\bar{b}$; (6) $a=b$.

Remark that the action $S(U)$ to $\delta_{a}$ corresponds to moving forward $\delta_{a}$ to its arcs whose origins are same as $t(a)$ except its inverse arc. Then the action $S(U)^{j}$ to $\delta_{a}$ corresponds to moving forward $\delta_{a}$ to $j$-step walks without any backtrackings. On the other hand, the action of $J$ corresponds to moving back to the inverse arc; that is, the backtrack. Then for cases (1)-(4), since ${ }^{\top} S(U)=J S(U) J$, it holds

$$
\left(S(U)^{j_{1}+1}\right)_{b, a}>0,\left(J S(U)^{j_{2}+1}\right)_{b, a}>0,\left({ }^{\top} S(U)^{j_{3}+1}\right)_{b, a}>0,\left(J^{\top} S(U)^{j_{4}+1}\right)_{b, a}>0,
$$

for cases (5) and $(6),(J)_{b, a}>0,(I)_{b, a}>0$, respectively. We should remark that there is a possibility that $\left(S(U)^{j_{1}+1}\right)_{b, a}>0$ and $\left(J S(U)^{j_{2}+1}\right)_{b, a}>0$, simultaneously since there might be a cycle which accomplishes both positional relations (1) and (2).

\section{$3.2 k$-regular tree case}

If the graph is a tree, the positional relation of arbitrary pair of arcs $(a, b)$ is uniquely applicable to one of the cases (1)-(6): if not, a cycle would appear in the tree. Note that the action of $S(U)^{j}$ to $\delta_{a}$ corresponds to $j$-walks without any backtracks while the action $J$ corresponds to the backtrack. Then if the graph is a tree, it holds

$$
\begin{gathered}
\left(S(U)^{j_{1}}\right)_{b, a}>0 \Leftrightarrow\left(S(U)^{j_{1}}\right)_{b, a}=1,{ }^{\forall} j \neq j_{1},\left(S(U)^{j}\right)_{b, a}=0 \\
\left(J S(U)^{j_{2}}\right)_{b, a}>0 \Leftrightarrow\left(J S(U)^{j_{2}}\right)_{b, a}=1,{ }^{\forall} j \neq j_{2},\left(J S(U)^{j}\right)_{b, a}=0 \\
\left(J^{\top} S(U)^{j_{3}}\right)_{b, a}>0 \Leftrightarrow\left(J^{\top} S(U)^{j_{3}}\right)_{b, a}=1,{ }^{\forall} j \neq j_{3},\left(J^{\top} S(U)^{j}\right)_{b, a}=0 \\
\left({ }^{\top} S(U)^{j_{4}}\right)_{b, a}>0 \Leftrightarrow\left({ }^{\top} S(U)^{j_{4}}\right)_{b, a}=1 .{ }^{\forall} j \neq j_{4},\left({ }^{\top} S(U)^{j}\right)_{b, a}=0
\end{gathered}
$$

We summarize the above observation as follows. 
(1)

$(2)$

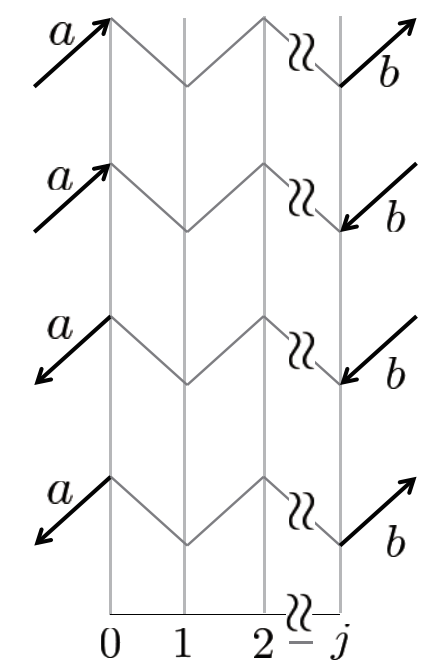

Figure 4: The positional relations of cases (1)-(4)

Lemma 6. Assume that the graph is a tree. Let $\Xi$ be the following set of matrices

$$
\left\{S(U)^{j}, J S(U)^{j}, J^{\top} S(U)^{j},{ }^{\top} S(U)^{j} \mid j \in \mathbb{N} \cup\{0\}\right\} .
$$

Then we have

$(a, b)$ is the positional relation (1)

$$
\Leftrightarrow{ }^{\exists} \text { s.t., }\left(S(U)^{j+1}\right)_{b, a}=1,{ }^{\forall} \xi \in \Xi \backslash\left\{S(U)^{j+1}\right\},(\xi)_{b, a}=0 ;
$$

$(a, b)$ is the positional relation (2)

$$
\Leftrightarrow{ }^{\exists} j \text { s.t. },\left(J S(U)^{j+1}\right)_{b, a}=1,{ }^{\forall} \xi \in \Xi \backslash\left\{J S(U)^{j+1}\right\},(\xi)_{b, a}=0 ;
$$

$(a, b)$ is the positional relation (3)

$$
\Leftrightarrow{ }^{\exists} \text { js.t., }\left({ }^{\top} S(U)^{j+1}\right)_{b, a}=1,{ }^{\forall} \xi \in \Xi \backslash\left\{{ }^{\top} S(U)^{j+1}\right\},(\xi)_{b, a}=0 ;
$$

$(a, b)$ is the positional relation (4)

$$
\Leftrightarrow{ }^{\exists} \text { s.t., }\left(J^{\top} S(U)^{j+1}\right)_{b, a}=1,{ }^{\forall} \xi \in \Xi \backslash\left\{J^{\top} S(U)^{j+1}\right\},(\xi)_{b, a}=0 ;
$$

$(a, b)$ is the positional relation (5)

$$
\Leftrightarrow(J)_{b, a}=1,{ }^{\forall} \xi \in \Xi \backslash\{J\},(\xi)_{b, a}=0 ;
$$

$(a, b)$ is the positional relation (6)

$$
\Leftrightarrow(I)_{b, a}=1,{ }^{\forall} \xi \in \Xi \backslash\{I\},(\xi)_{b, a}=0 .
$$

Let $\mathbb{T}$ be the $k$-regular tree (which is an infinite graph). From now on we fix an arbitrary arc $e$ as the initial arc and consider $S\left(U^{n}\right) \delta_{e}$. We take an isometric deformation of $\mathbb{T}$ so that we can keep the symmetricity with respect to this fixed arc $e$ : we "unbend" the tree by moving all the descendants of $o(e)$ to the opposite side as follows (see Fig 6): 
we decompose the vertices $V(\mathbb{T})$ into $\left(\ldots, V_{-1}, V_{0}, V_{1}, \ldots\right)$, where for $j \geqslant 1$,

$$
\begin{aligned}
V_{0} & =\{t(e)\}, \\
V_{-j} & =\{u \mid \operatorname{dist}(t(e), u)=j, \operatorname{dist}(o(e), u)=j-1\}, \\
V_{j} & =\{u \mid \operatorname{dist}(t(e), u)=j\} \backslash V_{-j}
\end{aligned}
$$

Remark that every vertex $u \in V_{j}(j \geqslant 1), \operatorname{dist}(o(e), u)=j+1$. We also decompose the arc set $A(\mathbb{T})$ into $\left(\ldots, A_{-1}^{R}, A_{0}^{R}, A_{1}^{R}, \ldots\right),\left(\ldots, A_{-1}^{L}, A_{0}^{L}, A_{1}^{L}, \ldots\right)$, where

$$
\begin{aligned}
& A_{j}^{R}=\left\{a \mid t(a) \in V_{j}, o(a) \in V_{j-1}\right\} ; \\
& A_{j}^{L}=\left\{a \mid t(a) \in V_{j}, o(a) \in V_{j+1}\right\} .
\end{aligned}
$$

Note that the positional relation between $a$ and $e$ is represented by $A_{j}^{N}$. By Lemma 6 , the matrix satisfying with $(\xi)_{a, e}=1$ is uniquely determined and represented by

$$
\xi= \begin{cases}S(U)^{j} & : a \in A_{j}^{R}(j \geqslant 0) ; \\ J S(U)^{j+1} & : a \in A_{j}^{L}(j \geqslant 0) ; \\ \top S(U)^{|j|} & : a \in A_{j}^{R}(j<0) ; \\ J^{\top} S(U)^{|j+1|} & : a \in A_{j}^{L}(j<0) .\end{cases}
$$

Figure 5 depicts a simple chart of this one-to-one correspondence between $\mathbb{Z} \times\{L, R\}$ and $\Xi$. Note that if $a, b \in A_{j}^{N}$, then $(\xi)_{a, e}=(\xi)_{b, e}$ for every $\xi \in \Xi$. On the other hand, the

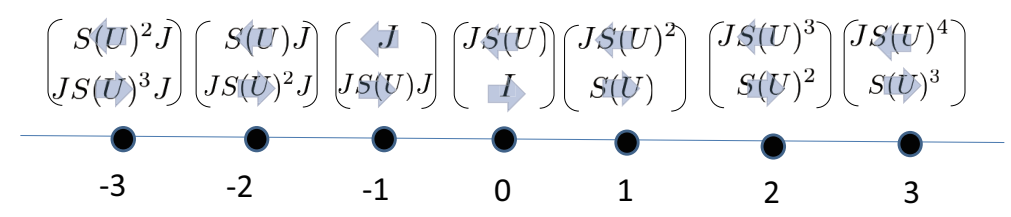

Figure 5: The one-to-one correspondence between $\mathbb{Z} \times\{L, R\}$ and $\Xi$ : The right directed arrow on the position $j \in \mathbb{Z}$ depicts $(j ; R)$, that is, $A_{j}^{R}$, and the left directed arrow on $j$ depicts $(j ; L)$, that is, $A_{j}^{L}$. The corresponding element of $\Xi$ to each arrow overlaps in this figure, e.g., the arrow $(2 ; L)$ corresponds to $J S(U)^{3}$.

following lemma shows that the amplitudes of the Grover walk itself; that is, $\left(U^{m}\right)_{a, e}$ and $\left(U^{m}\right)_{b, e}$ for $a, b \in A_{j}^{N}$, take also the same value.

Lemma 7. Assume $G$ is the k-regular tree. Let $\psi_{n}=U^{n} \delta_{e}$. Then

$$
\psi_{n}(a)=\psi_{n}(b)
$$

holds for any $a, b \in A_{j}^{N}$ with $j \in \mathbb{Z}$ and $N \in\{L, R\}$. 
Proof. For $n=0,(14)$ obviously holds. Assume (14) holds until $n$-step. Remark that by this assumption, if $a^{\prime} \in A_{j}^{(N)}$, we can put $\psi_{n}\left(a^{\prime}\right)=\tilde{\psi}_{n}(j ; N)$. Note that $\tilde{\psi}_{n}$ is a constant vector depended on only $j$ and $N$. From the definition of the Grover walk, we have

$$
\psi_{n+1}(a)=\left(U \psi_{n}\right)(a)=-\psi_{n}(\bar{a})+\frac{2}{k} \sum_{t(b)=o(a)} \psi_{n}(b) .
$$

If $a \in A_{j}^{(R)}$ and $j \geqslant 1$, then RHS is reexpressed by

$$
\begin{aligned}
R H S & =-\tilde{\psi}_{n}(j-1 ; L)+\frac{2}{k}\left(\sum_{b \in A_{j-1}^{(R)}, t(b)=o(a)} \psi_{n}(b)+\sum_{b \in A_{j-1}^{(L)}, t(b)=o(a)} \psi_{n}(b)\right) \\
& =-\tilde{\psi}_{n}(j-1 ; L)+\frac{2}{k}\left(\tilde{\psi}_{n}(j-1 ; R)+(k-1) \tilde{\psi}_{n}(j-1 ; L)\right)
\end{aligned}
$$

Since the expression is determined by only $j$ and $R$, we have $\psi_{n+1}(a)=\psi_{n+1}(b)$ for any $a, b \in A_{j}^{(R)}$. In the same way, we can show the statement for $a, b \in A_{j}^{(R)}(j \leqslant 0)$ case. Furthermore, we can show that $\psi_{n}(a)=\psi_{n}(b)$ for any $a, b \in A_{j}^{L}$.

Thus by (13) and Lemma $7, S\left(U^{n}\right) \delta_{e}$ is expressed by a linear combination of $\Xi \delta_{e}:=$ $\left\{\xi \delta_{e} \mid \xi \in \Xi\right\}$ with the $\{0,1\}$-coefficient; that is,

$$
S\left(U^{n}\right) \delta_{e}=\sum_{j=0}^{n}\left(\epsilon_{j} S(U)^{j} \delta_{e}+\tau_{j} J S(U)^{j} \delta_{e}\right)+\sum_{j=1}^{n-1}\left(\epsilon_{-j}^{\top}\left(S(U)^{j}\right) \delta_{e}+\tau_{-j} J^{\top}\left(S(U)^{j}\right) \delta_{e}\right),
$$

where $\epsilon_{j}, \tau_{j} \in\{0,1\}$. Moreover to obtain the $\{0,1\}$ coefficient of each term of $\Xi$, by Lemma 7, we pick up the parity of only the representative of each $A_{j}^{N}$ 's. To this end more efficiently, we introduce the following map: $\Psi: \ell^{2}(A(\mathbb{T})) \rightarrow \ell^{2}(\mathbb{Z} \times\{L, R\})$ such that

$$
(\Psi(\psi))(j ; R)=\frac{1}{\sqrt{\left|A_{j}^{R}\right|}} \sum_{a \in A_{j}^{R}} \psi(a), \quad(\Psi(\psi))(j ; L)=\frac{1}{\sqrt{\left|A_{j}^{L}\right|}} \sum_{a \in A_{j}^{L}} \psi(a) .
$$

We will regard $\Psi\left(\psi_{n}\right)(j ; N)$ as the "representative" of $\psi_{n}(a)$ for $a \in A_{j}^{N}$. A direct computation provides the following lemma.

Lemma 8. Let $\Psi$ and $\psi_{n}$ be the above. Then we have

$$
\begin{aligned}
& \left(\Psi\left(\psi_{n}\right)\right)(j ; R)= \begin{cases}\frac{2}{k} \sqrt{k-1}\left(\Psi\left(\psi_{n-1}\right)\right)(j-1 ; R)-\left(\frac{2}{k}-1\right)\left(\Psi\left(\psi_{n-1}\right)\right)(j-1 ; L) & : j \geqslant 0, \\
\frac{2}{k} \sqrt{k-1}\left(\Psi\left(\psi_{n-1}\right)\right)(j-1 ; R)+\left(\frac{2}{k}-1\right)\left(\Psi\left(\psi_{n-1}\right)\right)(j-1 ; L) & : j<0,\end{cases} \\
& \left(\Psi\left(\psi_{n}\right)(j ; L)= \begin{cases}\left(\frac{2}{k}-1\right)\left(\Psi\left(\psi_{n-1}\right)\right)(j+1 ; R)+\frac{2}{k} \sqrt{k-1}\left(\Psi\left(\psi_{n-1}\right)\right)(j+1 ; L) & : j \geqslant 0, \\
-\left(\frac{2}{k}-1\right)\left(\Psi\left(\psi_{n-1}\right)\right)(j+1 ; R)+\frac{2}{k} \sqrt{k-1}\left(\Psi\left(\psi_{n-1}\right)\right)(j+1 ; L) & : j<0 .\end{cases} \right.
\end{aligned}
$$


Putting $\phi_{n}(j)=^{\top}\left[\left(\Psi\left(\psi_{n}\right)\right)(j ; L) \quad\left(\Psi\left(\psi_{n}\right)\right)(j ; R)\right]$, we obtain the difference equation of the discriminant quantum walk; that is,

$$
\begin{aligned}
\phi_{0}(j) & =\delta_{0}(j)|R\rangle, \\
\phi_{n+1}(j) & =P(j+1) \phi_{n}(j+1)+Q(j-1) \phi_{n}(j-1),
\end{aligned}
$$

where $P(j)$ and $Q(j)$ are given by Definition 4 .

Remark 9. Let $\psi_{n}$ be the $n$-th iteration of the Grover walk on $k$-regular tree starting from $\delta_{e}$, and let $\phi_{n}$ be its discriminant quantum walk at time $n$. Then

$$
\psi_{n}(a)= \begin{cases}\frac{1}{\sqrt{\left|A_{j}^{R}\right|}}\left\langle R, \phi_{n}(j)\right\rangle & : a \in A_{j}^{R}, \\ \frac{1}{\sqrt{\left|A_{j}^{L}\right|}}\left\langle L, \phi_{n}(j)\right\rangle & : a \in A_{j}^{L} .\end{cases}
$$

This implies the phase is invariant with respect to $\Psi$.

Therefore by (13) and Remark 9 , it holds in the $k$-regular tree case that the coefficients in (15) are given by

$$
\left\langle R, \phi_{n}(j)\right\rangle>0 \Leftrightarrow \epsilon_{j}=1,\left\langle L, \phi_{n}(j)\right\rangle>0 \Leftrightarrow \tau_{j-1}=1 .
$$

Since $e \in A(\mathbb{T})$ can be chosen as an arbitrary arc due to the symmetricity of the $k$-regular tree, the statement of (19) also holds if we choose another initial arc $f \in A(\mathbb{T}) \backslash\{e\}$ and consider $S\left(U^{n}\right) \delta_{f}$ in the same way; which implies

$$
\begin{aligned}
& S\left(U^{n}\right)\left[\delta_{e}, \delta_{f}, \ldots\right] \\
& \quad=\left(\sum_{j=0}^{n}\left(\epsilon_{j} S(U)^{j}+\tau_{j} J S(U)^{j}\right)+\sum_{j=1}^{n-1}\left(\epsilon_{-j}^{\top}\left(S(U)^{j}\right)+\tau_{-j} J^{\top}\left(S(U)^{j}\right)\right)\right)\left[\delta_{e}, \delta_{f}, \ldots\right] .
\end{aligned}
$$

Since $\left[\delta_{e}, \delta_{f}, \ldots\right]=I$ with some appropriate computational basis order, the statement of our main theorem is true for the $k$-regular tree.

\section{$3.3 k$-regular graph with $g(G)>2(n-1)$ case}

We consider a $k$-regular graph whose girth is greater than $2(n-1)$. For an arbitrary fixed $e \in A(G)$, we put $t(e):=o$ and define $A_{i n}$ by the following sub-arc set such that

$$
A_{\text {in }}=\{a \in A \mid \operatorname{dist}(o, t(a)) \leqslant n-1, \operatorname{dist}(o, o(a)) \leqslant n-2\}
$$

Next we also define the boundary arc-set with respect to $A_{i n}$ as follows:

$$
\begin{gathered}
\partial A=\{a \in A \mid \operatorname{dist}(o, t(a))=n, \operatorname{dist}(o, o(a))=n-1\} \\
\cup\left\{a^{\prime}, a^{\prime \prime} \mid a \in A, \operatorname{dist}(o, t(a))=n-1, \operatorname{dist}(o, o(a))=n-1\right\} .
\end{gathered}
$$


If $a \in \partial A$ with $\operatorname{dist}(o, t(a))=\operatorname{dist}(o, o(a))(=n-1)$, then we "cut" the edge $|a|$ into $a^{\prime}$ and $a^{\prime \prime}$ such that $o\left(a^{\prime}\right)=o(a), o\left(a^{\prime \prime}\right)=o(\bar{a})$; but we regard $t\left(a^{\prime}\right), t\left(a^{\prime \prime}\right)=$ " $\varnothing$ ". See also Fig 6. The directed subgraph $\mathbb{T}_{e}^{(n)}=\left(V^{\prime}, A^{\prime}\right) \subset G$ is denoted by

$$
A^{\prime}=A_{\text {in }} \cup \partial A, \quad V^{\prime}=t\left(A_{\text {in }}\right)=\left\{t(a) \mid a \in A_{\text {in }}\right\},
$$

Note that in this setting, we omit the terminal vertices of all $a \in \partial A$.

Lemma 10. Let $G$ be a k-regular and $g(G)>2(n-1)$. Then $\mathbb{T}_{e}^{(n)}$ is a directed tree with depth $n$ and the root $t(e)$.

Proof. If there is a cycle in $\mathbb{T}_{e}^{(n)}$, then the cycle does not pass any arcs in $\partial A$ since all the terminal vertices $\partial A$ are vanished. The length of a largest cycle in this graph is not less than the girth of $G$, that is $2(n-1)+1$, which should run through each level set. To accomplish this cycle, we need the arc connecting two vertices in $(n-1)$-level set, but such an arc belongs to non available arcs in $\partial A$ by the definition. Then the largest length of the cycle is at most $2(n-1)$. By the assumption $g(G)>2(n-1)$, then the contradiction occurs. Thus there are no cycles.

Thus for the $k$-regular graph $G$ whose girth is greater than $2(n-1)$, when we look around from an arbitrary vertex, we can regard it as a local $k$-regular tree within $(n-1)$ distance from the vertex. For a graph $H$, the time evolution of the Grover walk on the graph $H$ is denoted by $U_{H}$. By Lemma 10, the following statement is immediately obtained.

Lemma 11. Let $G$ be k-regular with $g(G)>2(n-1)$ and also let $\mathbb{T}_{e}^{(n)}$ be the above directed subtree with depth $n$. Then for every $e \in A(G)$, and for every $f \in A^{\prime}$,

$$
\left(U_{G}^{j}\right)_{f, e}=\left(U_{\mathbb{T}_{e}^{(n)}}^{j}\right)_{f, e}(j=0,1, \ldots, n) .
$$

Proof. If $f \in A_{\text {in }}$, the statement is trivial. We show it for $f \in \partial A$ case. To reach $f \in \partial A$ from the initial arc $e$, it takes at least $n$ iterations. If there are two shortest paths from $e$ to $f ;\left(e, e_{1}, \ldots, e_{n-1}, f\right)$ and $\left(e, e_{1}^{\prime}, \ldots, e_{n-1}^{\prime}, f\right)$, then since $o\left(e_{1}\right)=o\left(e_{1}^{\prime}\right)=t(e)$ and $t\left(e_{n-1}\right)=t\left(e_{n-1}^{\prime}\right)=o(f)$, the $2(n-1)$-length cycle appears. This is contradiction to the assumption $g(G)>2(n-1)$.

Therefore when the Grover walk on the $k$-regular graph $G$; whose girth is greater than $2(n-1)$, starts from $\delta_{e}$, we can convert it to the Grover walk on the $k$-regular tree starting from $e$ whose terminus is the root of the tree as long as the time iteration is less than $n$. Since $e \in A(G)$ can be chosen as an arbitrary arc, the same statement also holds if we choose another initial arc $e^{\prime} \in A(G) \backslash\{e\}$ and consider $S\left(U^{n}\right) \delta_{e^{\prime}}$. It completes the proof. 

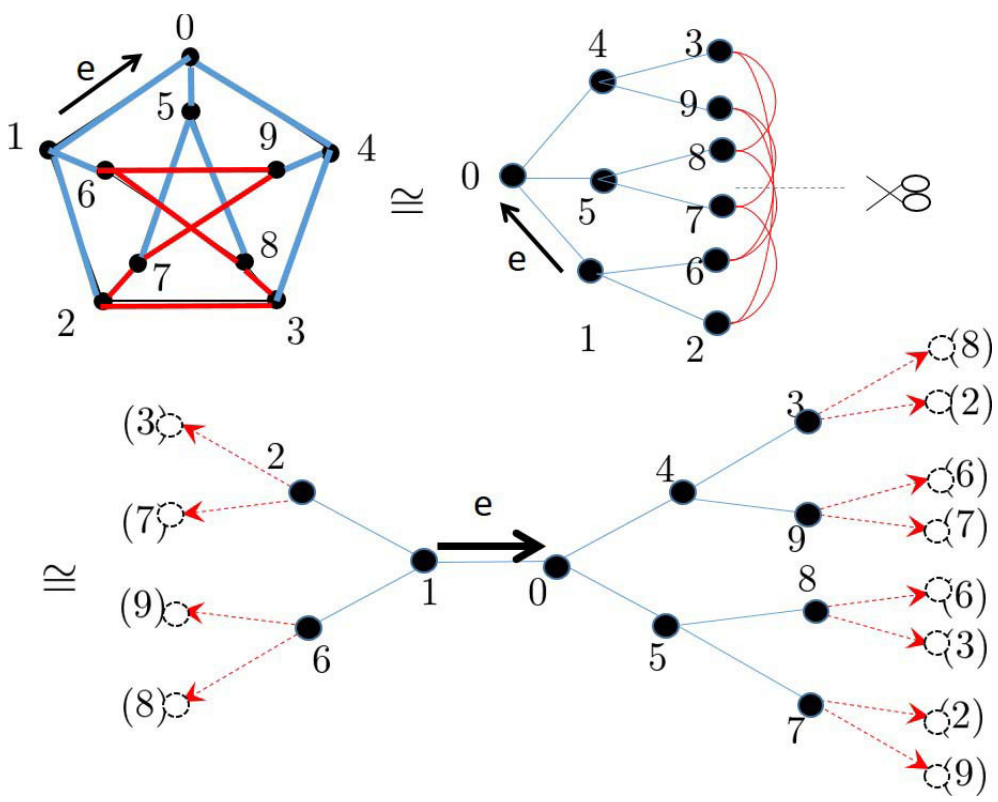

Figure 6: The idea of the main theorem: Let us start the walk from the arc $e$ of the Petersen graph in this figure $(g(G)=5)$. After taking an automorphism transpose with respect to the distance from $t(e)$, we cut the red edges. We take an automorphism transpose to this tree again so that the subtree whose vertices are descendants of $o(e)$ is placed to the opposite side. By using this symmetricity of the last figure, we can show that this Grover walk is essentially same as the discriminant quantum walk until $(n=) 3$-step.

\section{Spectrum of $S\left(U^{n}\right)$}

For given $k$-regular graph $G=(V, E)$, we consider the spectrum of $S\left(U^{n}\right)$. We show the spectrum of $S\left(U^{n}\right)$ is inherited from the spectrum of the underlying graph. Although the setting of [22], whose setting was for an extended Szegedy walk, is different from our setting, our fundamental analytical method can follow [22] with some modifications to be able to apply to our setting. The operation " $S$ " will remove regularities of the unitary operator $U^{n}$. This method enables us to find when $S\left(U^{n}\right)$ loses the diagonalizable property.

We introduce $K: \ell^{2}(A) \rightarrow \ell^{2}(V)$ as the following boundary operator corresponding to an incidence matrix with respect to the terminal vertices:

$$
K=\sum_{a \in A}|t(a)\rangle\langle a|
$$

where $|u\rangle$ and $|a\rangle$ denote the standard basis of $\ell^{2}(V)$ and $\ell^{2}(V)$ corresponding to $u \in V$, $a \in A$, respectively. This boundary operator $K$ plays the important role for our spectral analysis due to the following properties:

1. $S(U)=J\left(K^{*} K-I\right)$; 
2. $K K^{*}=k \mathbf{1}_{|V|}$;

3. $K J K^{*}=M$, where $M$ is the adjacency matrix of $G$.

We put $L: \ell^{2}(V) \times \ell^{2}(V) \rightarrow \ell^{2}(A)$ such that $L=\left[K^{*}, J K^{*}\right]$. Using the above properties of $K^{*}$ and $J^{2}=\mathbf{1}_{|A|}$, we obtain

$$
S(U) L=L \tilde{M}
$$

Here $\tilde{M}: \ell^{2}(V) \times \ell^{2}(V) \rightarrow \ell^{2}(V) \times \ell^{2}(V)$ is

$$
\tilde{M}=\left[\begin{array}{cc}
0 & -1 \\
k-1 & M
\end{array}\right] .
$$

Remarking that ${ }^{\top} S(U)=J S(U) J$, we can easily obtain that

$$
\begin{aligned}
S(U)^{j} L & =L \tilde{M}^{j} ; \\
J S(U)^{j} L & =L \tilde{\sigma}_{X} \tilde{M}^{j} ; \\
{ }^{\top} S(U)^{j} L & =L \tilde{\sigma}_{X} \tilde{M}^{j} \tilde{\sigma}_{X} ; \\
J^{\top} S(U)^{j} L & =L \tilde{M}^{j} \tilde{\sigma}_{X} .
\end{aligned}
$$

Here $\tilde{\sigma}_{X}=\sigma_{X} \otimes I_{|V|}$ with

$$
\sigma_{X}=\left[\begin{array}{ll}
0 & 1 \\
1 & 0
\end{array}\right]
$$

Let $F(x ; y)$ be a linear combination of $\left\{x^{j}, y x^{j}, y x^{j} y, x^{j} y \mid j \in \mathbb{Z}_{\geqslant 0}\right\}$. Then by (21),

$$
F(S(U) ; J) L=L F\left(\tilde{M} ; \tilde{\sigma}_{X}\right) .
$$

Let $\mathcal{L} \subset \ell^{2}(A)$ be $L\left(\ell^{2}(V) \times \ell^{2}(V)\right)$, that is, $\mathcal{L}=\left\{K^{*} f+J K^{*} g \mid f, g \in \ell^{2}(V)\right\}$. By $(22)$, we have the following equivalent deformation with respect to the eigenequation of $F(S(U) ; J)$ restricted to $\mathcal{L}$;

$$
\left.F(S(U) ; J)\right|_{\mathcal{L}} \psi=\lambda \psi, \quad \psi \neq 0 \Leftrightarrow L\left(\lambda-F\left(\tilde{M} ; \tilde{\sigma}_{X}\right)\right) \phi=0, \quad \phi \notin \operatorname{ker} L .
$$

Thus we need to clarify ker $L$. Indeed this is expressed as follows.

Lemma 12. Let $L$ and $\tilde{M}$ be as the above. Then we have

$$
\operatorname{ker}(L)=\operatorname{ker}\left(1-\tilde{M}^{2}\right)=\operatorname{ker}\left(k+\left[\begin{array}{cc}
0 & M \\
M & 0
\end{array}\right]\right) .
$$

Proof. First we show $\operatorname{ker}(L) \subset \operatorname{ker}\left(1-\tilde{M}^{2}\right)$. Let ${ }^{\top}[f, g] \in \operatorname{ker}(L)$. Then $K^{*} f+J K^{*} g=0$ holds. By multiplying $K$ and $K J$, then we have

$$
k f+M g=0, \quad M f+k g=0 .
$$

Using this equation, a simple computation leads

$$
\left(1-\tilde{M}^{2}\right)^{\top}[f, g]=0 .
$$


which implies ${ }^{\top}[f, g] \in \operatorname{ker}\left(1-\tilde{M}^{2}\right)$, that is, $\operatorname{ker}(L) \subset \operatorname{ker}\left(1-\tilde{M}^{2}\right)$. Next we show $\operatorname{ker}(L) \supset \operatorname{ker}\left(1-\tilde{M}^{2}\right)$. We assume ${ }^{\top}[f, g] \in \operatorname{ker}\left(1-\tilde{M}^{2}\right)$. Then

$$
k f+M g=0, M f+k g=0 .
$$

Remarking $K K^{*}=k$ and $K J K^{*}=M$, we can provides the following equivalent expression of the above equations:

$$
K\left(K^{*} f+J K^{*} g\right)=0, K J\left(K^{*} f+J K^{*} g\right)=0,
$$

which are also equivalent to $K^{*} f+J K^{*} g \in \mathcal{L}^{\perp}$. Thus $K^{*} f+J K^{*} g$ should be 0 since $K^{*} f+J K^{*} g \in \mathcal{L}$. It is completed the proof.

Since the adjacency matrix $M$ is a regular matrix, then $M$ can be decomposed into

$$
M=\sum_{\mu \in \sigma(M)} \mu \Pi_{\mu}
$$

where $\Pi_{\mu}$ is the orthogonal projection onto the eigenspace of $\mu$ and $\sigma(M)$ is set of all eigenvalues of $M$. Remarking $\sum_{\mu} \Pi_{\mu}=I_{|V|}$, we can decompose $\tilde{M}$ into

$$
\tilde{M}=\sum_{\mu \in \sigma(M)}\left[\begin{array}{cc}
0 & -1 \\
k-1 & \mu
\end{array}\right] \otimes \Pi_{\mu} .
$$

Therefore the equivalent deformations of the eigenequation (23) can be continued to

$$
\begin{aligned}
\left(1-\tilde{M}^{2}\right)(\lambda & \left.-F\left(\tilde{M} ; \tilde{\sigma}_{X}\right)\right) \phi=0, \quad \phi \notin \operatorname{ker}\left(1-\tilde{M}^{2}\right) \\
& \Leftrightarrow\left(\sum_{\mu \in \sigma(M)}\left(1-K_{\mu}^{2}\right)\left(\lambda-F\left(K_{\mu} ; \sigma_{X}\right)\right) \otimes \Pi_{\mu}\right) \phi=0, \quad \phi \notin \operatorname{ker}\left(1-\tilde{M}^{2}\right)
\end{aligned}
$$

Here $K_{\mu}$ is the 2-dimensional matrix defined by

$$
K_{\mu}=\left[\begin{array}{cc}
0 & -1 \\
k-1 & \mu
\end{array}\right]
$$

We are interested in so-called non-trivial zeros ( or eigenvalues) of

$$
\operatorname{det}\left(\lambda-S\left(U^{n}\right)\right)=0
$$

not living in the real line, which is a graph analogue of the non-trivial poles of the Riemann zeta function; that is the zeros of the Ihara zeta $[2,15,21]$ motivated by the quantum walks. Remark that all the eigenvalues for the eigenspace $\mathcal{L}^{\perp}$ are included in $\mathbb{R}$ because $\mathcal{L}^{\perp}=\operatorname{ker}(K) \cap \operatorname{ker}(K J)=\{\operatorname{ker}(K) \cap \operatorname{ker}(1-J)\} \cap\{\operatorname{ker}(K) \cap \operatorname{ker}(1+J)\}$. Let $F_{n}(x ; y)$ 
be the linear combination of $\left\{x^{j}, y x^{j}, y x^{j} y, x^{j} y \mid j \in \mathbb{Z}_{\geqslant 0}\right\}$ determined by (5) switching the notations of $S(U)$ and $J$ in (5) to $x$ and $y$, respevetively; that is,

$$
F_{n}(x ; y)=\sum_{j=0}^{n}\left(\epsilon_{j} x^{j}+\tau_{j} y x^{j}\right)+\sum_{j=1}^{n-1}\left(\epsilon_{-j} y x^{j} y+\tau_{-j} x^{j} y\right) .
$$

For example, if $n=3$, then $F_{3}(x ; y)=x^{3}+y x y$. Here $x$ and $y$ are non-comutative in general. ${ }^{1}$ Then to see the non-trivial eigenvalues, we can concentrate on the eigenequation:

Theorem 13. Let $\lambda$ be the non-trivial eigenvalue of $S\left(U^{n}\right)$. Then the value $\lambda$ satisfies

$$
\operatorname{det}\left(\lambda-F_{n}\left(K_{\mu} ; \sigma_{X}\right)\right)=0
$$

for some $\mu \in \sigma(M)$.

Proof. Let us consider the decomposition (25). We consider the solution of the eigenequation

$$
\operatorname{det}\left(\sum_{\mu \in \sigma(M)}\left(1-K_{\mu}^{2}\right)\left(\lambda-F_{n}\left(K_{\mu} ; \sigma_{X}\right)\right) \otimes \Pi_{\mu}\right)=0 .
$$

Let $\sigma(M)=\left\{\mu_{1}, \ldots, \mu_{|V|}\right\}$, and $A_{\mu_{j}}:=\left(1-K_{\mu_{j}}^{2}\right)\left(\lambda-F_{n}\left(K_{\mu_{j}} ; \sigma_{X}\right)\right)$ for $j=1, \ldots,|V|$. Remark that for any $2 \times 2$ matrix $X$ and $|V| \times|V|$ matrix $Y, X \otimes Y=Q^{*}(Y \otimes X) Q$ with $Q(\boldsymbol{x} \otimes \boldsymbol{y})=\boldsymbol{y} \otimes \boldsymbol{x}$ for $\boldsymbol{x} \in \mathbb{C}^{2}, \boldsymbol{y} \in \mathbb{C}^{|V|}$ and we also remark that $Q Q^{*}=Q^{*} Q=I_{2|V|}$. Moreover it holds $\Pi_{\mu_{j}}=(P|j\rangle)(P|j\rangle)^{*}$, where $P$ is the unitary matrix on $\mathbb{C}^{|V|}$ such that $P^{-1} M P=\operatorname{diag}\left[\mu_{1}, \ldots, \mu_{|V|}\right]$ and $|j\rangle$ is the standard base of $\mathbb{C}^{|V|}$ labeled by $j$. Then

$$
\sum_{j=1}^{|V|} A_{\mu_{j}} \otimes \Pi_{\mu_{j}}=\left(P^{*} \otimes I_{2}\right) Q^{*}\left(\sum_{j=1}^{|V|}|j\rangle\langle j| \otimes A_{\mu_{j}}\right) Q\left(P \otimes I_{2}\right)
$$

Therefore (28) is equivalent to

$$
\operatorname{det}\left[\begin{array}{cccc}
A_{\mu_{1}} & 0 & \cdots & 0 \\
0 & A_{\mu_{2}} & \cdots & 0 \\
\vdots & \vdots & \ddots & \vdots \\
0 & 0 & \cdots & A_{\mu_{|V|}}
\end{array}\right]=0
$$

The eigenequation can be reduced as follows:

$$
\prod_{\mu \in \sigma(M)} \operatorname{det}\left(1-K_{\mu}^{2}\right)\left(\lambda-F_{n}\left(K_{\mu} ; \sigma_{X}\right)\right)=0
$$

First we easily notice that $\operatorname{det}\left(1-K_{\mu}^{2}\right)=0$ iff $\mu= \pm k$. Then if $\mu \neq \pm k$, the solution of $\operatorname{det}\left(\lambda-F_{n}\left(K_{\mu} ; \sigma_{X}\right)\right)=0$ with respect to $\lambda$ is an eigenvalue of $F_{n}(S(U) ; J)=S\left(U^{n}\right)$.

\footnotetext{
${ }^{1}$ We used ${ }^{\top} S(U)=J S(U) J$ and $J^{\top} S(U)=S(U) J$ for $(26)$.
} 
Then we showed that the statement is true at least for $\mu \neq \pm k$. So secondly we consider $\mu=k$ case. Since

$$
\operatorname{ker}\left(1-K_{k}^{2}\right)=\operatorname{ker}\left[\begin{array}{ll}
k & k \\
k & k
\end{array}\right]
$$

we have

$$
\operatorname{ker}\left(1-K_{k}^{2}\right)\left(\lambda-F_{n}\left(K_{k} ; \sigma_{X}\right)\right)=\left\{\left[\begin{array}{l}
\alpha \\
\beta
\end{array}\right] \mid(a+c-\lambda) \alpha+(b+d-\lambda) \beta=0\right\}
$$

when we put

$$
F_{n}\left(K_{k} ; \sigma_{X}\right)=\left[\begin{array}{cc}
a & b \\
c & d
\end{array}\right]
$$

Moreover it is easy to compute that

$$
K_{k}^{j}=\frac{1}{k-2}\left[\begin{array}{cc}
(k-1)-(k-1)^{j} & 1-(k-1)^{j} \\
(k-1)^{j+1}-(k-1) & -1+(k-1)^{j+1}
\end{array}\right] .
$$

Let

$$
K_{k}^{j}=\frac{1}{k-2}\left[\begin{array}{ll}
p & q \\
r & s
\end{array}\right]
$$

Then we have $p+r=q+s$. Furthermore, we have

$$
K_{k}^{j} \sigma_{X}=\frac{1}{k-2}\left[\begin{array}{ll}
q & p \\
s & r
\end{array}\right], \sigma_{X} K_{k}^{j}=\frac{1}{k-2}\left[\begin{array}{ll}
r & s \\
p & q
\end{array}\right],
$$

which implies $a+c=b+d$. Then if $a+c-\lambda \neq 0$, then

$$
\operatorname{ker}\left(1-K_{k}^{2}\right)\left(\lambda-F_{n}\left(K_{k} ; \sigma_{X}\right)\right)=\left\{\left[\begin{array}{l}
\alpha \\
\beta
\end{array}\right] \mid \alpha+\beta=0\right\}
$$

However in that case, we can check that $\left[\begin{array}{l}\alpha \\ \beta\end{array}\right] \in \operatorname{ker}\left(1-\tilde{M}^{2}\right)$. Thus $\lambda=a+c$. which is a real value and not a non-trivial eigenvalue. The case for $\mu=-k$ can be done in the same way as $\mu=k$ case. Therefore $\mu= \pm k$ cases can be excluded.

Theorem 5 shows the concrete expression of $F_{n}\left(K_{\mu}, \sigma_{X}\right)$ for each $n$. For example, for $n=3$ case, $(27)$ is reduced to

$$
\operatorname{det}\left(\lambda-\left(K_{\mu}^{3}+\sigma_{X} K_{\mu} \sigma_{X}\right)\right)=0
$$

We put the solution $\lambda(\mu):=x(\mu)+\mathbf{i} y(\mu)$ with $x(\mu), y(\mu) \in \mathbb{R}$. Since $F_{n}(\mu):=F_{n}\left(K_{\mu} ; \sigma_{X}\right)$ is a $2 \times 2$ matrix, the solution of $(27)$ with respect to $\lambda$ is that of the following characteristic polynomial:

$$
\lambda^{2}-\operatorname{tr}\left(F_{n}(\mu)\right) \lambda+\operatorname{det}\left(F_{n}(\mu)\right)=0
$$


Define $D_{n}(\mu):=\operatorname{tr}\left(F_{n}(\mu)\right)^{2}-4 \operatorname{det}\left(F_{n}(\mu)\right)$. The real part $x(\mu)$ of this solution $\lambda(\mu)$ lies on

$$
\begin{aligned}
R_{n}:=\{(\mu, x) \in[-k, k] & \left.\times \mathbb{R} \mid x^{2}-\operatorname{tr}\left(F_{n}(\mu)\right) x+\operatorname{det}\left(F_{n}(\mu)\right)=0, D_{n}(\mu) \geqslant 0\right\} \\
& \cup\left\{(\mu, x) \in[-k, k] \times \mathbb{R} \mid \operatorname{tr}\left(F_{n}(\mu)\right)-2 x=0, D_{n}(\mu) \leqslant 0\right\} .
\end{aligned}
$$

On the other hand, the imaginary part $y(\mu)$ lies on the following algebraic equation which draws a kind of hyperelliptic curve for $n \geqslant 2$ :

$$
I_{n}:=\left\{(\mu, y) \in[-k, k] \times \mathbb{R} \mid 4 y^{2}+D_{n}(\mu)=0\right\} .
$$

Therefore the non-trivial eigenvalues of $S\left(U^{n}\right)$ are inherited from all the eigenvalues of the underlying graph satisfying $D_{n}(\mu) \leqslant 0$. In Appendix, explicit expressions for $R_{n}(\mu)$ and $I_{n}(\mu)$ for $n=1, \ldots, 6$ are described. The parity of $D_{n}(\cdot)$ determines the range of the spectrum of $S\left(U^{n}\right)$. On the other hand, the following theorem shows the affect of zero's of $D_{n}(\cdot)$ on the matrix property of $S\left(U^{n}\right)$.

Theorem 14. A solution for $D_{n}(\mu)=0 ; \mu_{*}$, belongs to $\sigma(M)$ and $F_{n}\left(\mu_{*}\right) \neq c I$ with some nonzero constant $c$ if and only if $S\left(U^{n}\right)$ is not diagonalizable.

Proof. This is obtained by the fact that the geometric multiplicity of the eigenvalue $\lambda\left(\mu_{*}\right)$ of $K_{\mu_{*}}$ is strictly less than the algebraic multiplicity: If $\operatorname{ker}\left(\lambda-F_{n}(\mu)\right) \subset \operatorname{ker}\left(\lambda-F_{n}(\mu)\right)^{2}$, then $D_{n}(\mu)=0$ since the solution for $\lambda^{2}-\operatorname{tr}\left(F_{n}(\mu)\right) \lambda+\operatorname{det}\left(F_{n}(\mu)\right)=0$ must have a multiple root. Remark that if $D_{n}(\mu)=0$ with $\operatorname{ker}\left(\lambda-F_{n}(\mu)\right)=\operatorname{ker}\left(\lambda-F_{n}(\mu)\right)^{2}$, then $F_{n}(\mu)=c I$ with some constant $c$ since $F_{n}(\mu)$ is a $2 \times 2$ matrix. Therefore $\operatorname{ker}\left(\lambda-F_{n}(\mu)\right) \subset$ $\operatorname{ker}\left(\lambda-F_{n}(\mu)\right)^{2}$ if and only if $D_{n}(\mu)=0$ and $F_{n}(\mu) \neq c I$.

Remark that if the spectrum of the underlying graph has the branch points of $R_{n}$ (see Fig 8), then

$$
\sigma(M) \cap\left\{\mu \mid D_{n}(\mu)=0\right\} \neq \varnothing .
$$

If such a $\mu_{*} \in\left\{\mu \in \sigma(M) \mid D_{n}(\mu)=0\right\}$ satisfies with $F_{n}\left(\mu_{*}\right) \neq c I$, then $S\left(U^{n}\right)$ becomes non-diagonalizable.

Finally we draw the support of non-trivial eigenvalues with the parameter $\mu \in[-k, k] \supset$ $\sigma(M)$ in the complex plane, that is, $\left\{(x(\mu), y(\mu)) \mid \mu \in[-k, k], D_{n}(\mu) \leqslant 0\right\}$ in Fig. 7 and $I_{n}$ and $R_{n}$ to show how $\Re\left(\sigma\left(S(U)^{n}\right)\right)$ and $\Im\left(\sigma\left(S(U)^{n}\right)\right)$ are inherited from $\sigma(M)$ in Fig. 8 for $n=1,2, \ldots, 6$.

\section{Summary}

We obtained the structure theorem of the $n$-th power of the time evolution of the Grover walk on $G$ (Theorem 5) and its support of the non-trivial spectrum for the girth $g(G)>$ $2(n-1)$ (Theorem 13). The non-trivial spectrum is not living on the real line, which is a graph analogue of the non-trivial poles of the Riemann zeta function. We showed that this problem is converted to solving the phase pattern of the one-dimensional quantum walk in Definition 4 which is only determined by the regularity of $G$. The curious phase pattern can be seen in Fig. 3 and the support of the spectrum can be seen in Fig. 4 . Solving rigorously this phase pattern is one of the interesting future's problems. 


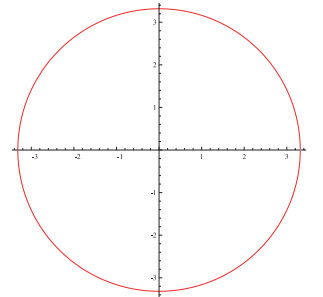

(1) The support of eigenvalue of $S(U)$

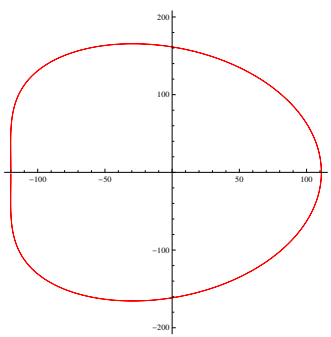

(4) The support of eigenvalue of $S\left(U^{4}\right)$

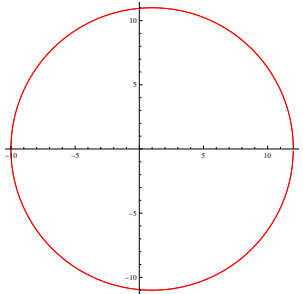

(2) The support of eigenvalue of $S\left(U^{2}\right)$

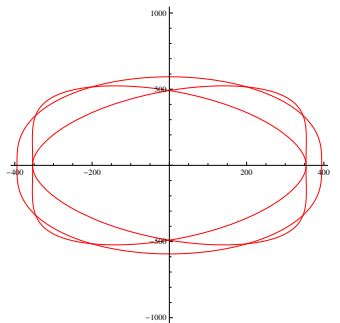

(5) The support of eigenvalue of $S\left(U^{5}\right)$

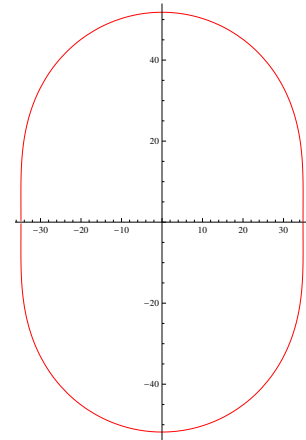

(3) The support of eigenvalue of $S\left(U^{3}\right)$

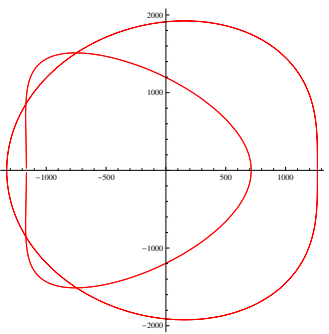

(6) The support of eigenvalue of $S\left(U^{6}\right)$

Figure 7: Figures (1)-(6) are the support of the non-trivial eigenvalues in $\mathbb{C}$ of $S(U), S\left(U^{2}\right), \ldots, S\left(U^{6}\right)$, respectively for $k=12$ and $g(G)>2(n-1)$. The horizontal and vertical lines are real and imaginary lines, respectively. The eigenvalues of $S\left(U^{n}\right)$ must lie on this support for each $n$.

\section{Appendix A}

The curve $R_{n}(\mu)$ for $D_{n}(\mu) \leqslant 0$ is the set of the zero's of the following polynomial $Q_{n}$ with respect to $\mu$ and $x$ :

$$
\begin{gathered}
Q_{1}=\mu-2 x \\
Q_{2}=(4-2 k)+\mu^{2}-2 x \\
Q_{3}=(4-3 k) \mu+\mu^{3}-2 x \\
Q_{4}=\left(6-6 k+2 k^{2}\right)+(5-4 k) \mu^{2}+\mu^{4}-2 x \\
Q_{5}=\left(6-11 k+5 k^{2}\right) \mu+(6-5 k) \mu^{3}+\mu^{5}-2 x \\
Q_{6}=\left(4-6 k+6 k^{2}-2 k^{3}\right)+\left(10-20 k+9 k^{2}\right) \mu^{2}+(7-6 k) \mu^{4}+\mu^{6}-2 x
\end{gathered}
$$

The curve $I_{n}(\mu)$ is the set of the zero's of the following polynomial $P_{n}$ with respect to $\mu$ and $y$ which draws a hyperelliptic curve for $n \geqslant 2$, where $D_{n}(\mu)$ is obtained by $P_{n}-4 y^{2}$.

$$
\begin{gathered}
P_{1}=(4-4 k)+\mu^{2}+4 y^{2} \\
P_{2}=(4-4 k) \mu^{2}+\mu^{4}+4 y^{2}
\end{gathered}
$$




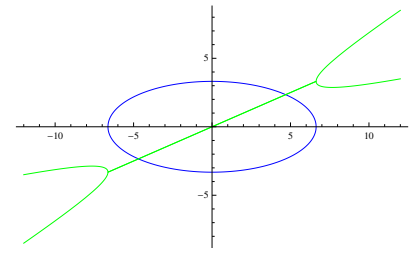

$I_{1}$ and $R_{1}$ for $k=12$

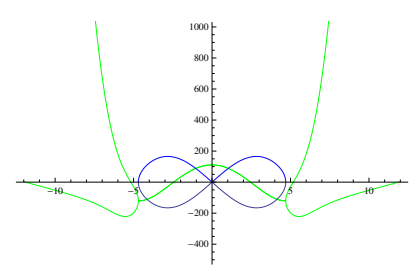

$I_{4}$ and $R_{4}$ for $k=12$

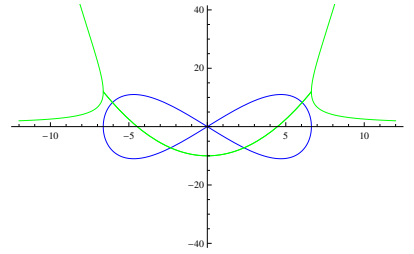

$I_{2}$ and $R_{2}$ for $k=12$

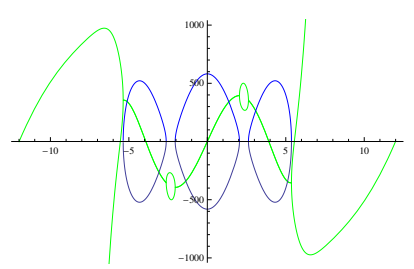

$I_{5}$ and $R_{5}$ for $k=12$

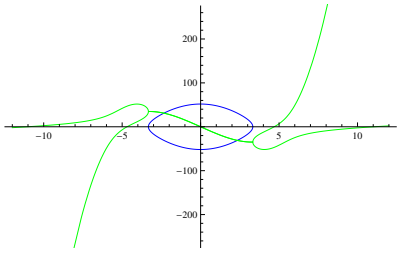

$I_{3}$ and $R_{3}$ for $k=12$

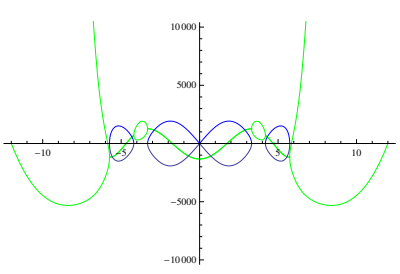

$I_{6}$ and $R_{6}$ for $k=12$

Figure 8: The orbits of real and imaginary parts of eigenvalue of $S\left(U^{n}\right)$ for $k=12$ and $g(G)>2(n-1)(n=1, \ldots, 6)$ obtained by (29) and (30), respectively: The horizontal line is the parameter $\mu \in[-k, k] \supset \sigma(M)$, and the vertical line is the real and imaginary parts of eigenvalue of $S\left(U^{n}\right) ; R_{n}(\mu)$ and $I_{n}(\mu)$, respectively. The green curve depicts $R_{n}(\mu)$ and the blue curve depicts $I_{n}(\mu)$. Thus $R_{n}(\mu)$ and $I_{n}(\mu)$ show how the spectrum of the graph is mapped to the real and imaginary parts of that of induced $S\left(U^{n}\right)$. If the spectrum of the graph has the branch points in $R_{n}(\mu)$, then $S\left(U^{n}\right)$ is non-diagonalizable.

$$
\begin{gathered}
P_{3}=-8\left(-2+4 k-3 k^{2}+k^{3}\right)+\left(16-24 k+13 k^{2}\right) \mu^{2}+(4-6 k) \mu^{4}+\mu^{6}+4 y^{2} \\
P_{4}=12\left(3-7 k+6 k^{2}-2 k^{3}\right) \mu^{2}+\left(25-44 k+24 k^{2}\right) \mu^{4}+(6-8 k) \mu^{6}+\mu^{8}+4 y^{2} \\
\begin{aligned}
& P_{5}=(16-48 k\left.+76 k^{2}-68 k^{3}+36 k^{4}-8 k^{5}\right)+\left(48-152 k+205 k^{2}-146 k^{3}+41 k^{4}\right) \mu^{2} \\
&+\left(52-156 k+174 k^{2}-66 k^{3}\right) \mu^{4}+\left(28-66 k+39 k^{2}\right) \mu^{6} \\
&+(8-10 k) \mu^{8}+\mu^{10}+4 y^{2} \\
& P_{6}=-12\left((-1+k)^{3}\left(3-7 k+5 k^{2}\right)\right) \mu^{2}+\left(100-428 k+704 k^{2}-524 k^{3}+149 k^{4}\right) \mu^{4} \\
&+\left(100-324 k+358 k^{2}-136 k^{3}\right) \mu^{6}+(45-\left.100 k+58 k^{2}\right) \mu^{8} \\
&+(10-12 k) \mu^{10}+\mu^{12}+4 y^{2}
\end{aligned}
\end{gathered}
$$

Remark that for $k \geqslant 5$, the forms of $\left\{Q_{n}\right\}$ and $\left\{P_{n}\right\}$ need classifications with respect to the value $k$; see (8)-(12); the above forms are in the case of $k \geqslant 12$. The forms of $Q_{n}$ and $P_{n}$ for general $n$ can be obtained by running the discriminant quantum walk on $\mathbb{Z}$ until $n$-step, and taking the phase measurement; e.g., for $k=20$, the phase pattern until $n=100$ can be referred in Fig. 2 .

\section{Acknowledgements}

E.S. acknowledges financial supports from the Grant-in-Aid of Scientific Research (C) Japan Society for the Promotion of Science (Grant No. 19K03616). 


\section{References}

[1] Ambainis, A.: Quantum walks and their algorithmic applications, Int. J. Quantum Inf. 1 (2003) pp.507-518.

[2] Bass, H.: The Ihara-Selberg zeta function of a tree lattice. Internat. J. Math. 3 (1992) pp.717-797

[3] Endo, T., Mohamed, S. and Segawa, E.: Phase measurement of quantum walks on the two-phase model, in preparation.

[4] Emms, D., Hancock, E. R., Severini, S., Wilson, R. C., A matrix representation of graphs and its spectrum as a graph invariant, Electr. J. Combin. 13 (2006) R34.

[5] Foata, D., Zeilberger, D.: A combinatorial proof of Bass's evaluations of the IharaSelberg zeta function for graphs, Trans. Amer. Math. Soc. 351 (1999) pp.2257-2274.

[6] Feynman, R. P., Hibbs, A. R.: Quantum Mechanics and Path Integrals, Dover Publications, Inc., Mineola, NY, emended edition, 2010.

[7] Godsil, C.: State transfer on graphs, Discrete Mathematics 312 (2011) pp.129-147.

[8] Godsil, C., Guo, K.: Quantum walks on regular graphs and eigenvalues. Electron. J. Combin. 18 (2011) R165.

[9] Godsil, C., Guo, K., Myklebust, Tor G.J.: Quantum walks on generalized quadrangles, The electronic journal of combinatorics 24 (2017) $\sharp \mathrm{P} 4.16$.

[10] Guo, K.: Quantum walks on strongly regular graphs. Master's thesis, University of Waterloo (2010).

[11] Hashimoto, K.: Zeta Functions of Finite Graphs and Representations of p-Adic Groups, In: "Adv. Stud. Pure Math." 15 pp.211-280, Academic Press, New York (1989)

[12] Higuchi, Yu., Konno, N., Sato, I., Segawa, E.: A note on the discrete-time evolutions of quantum walk on a graph. J. Math-for-Ind. 5B (2013) pp.103-109.

[13] Higuchi, Yu., Konno, N., Sato, I., Segawa, E.: Spectral and asymptotic properties of Grover walks on crystal lattices. J. Funct. Anal. 267 (2014) pp.4197-4235.

[14] Higuchi, Yu., Segawa, E.: Quantum walks induced by Dirichlet random walks on infinite trees, accepted for publication to Journal of Physics A: Mathematics and Theoretical 51 (2018) 075303.

[15] Ihara, Y.: On discrete subgroups of the two by two projective linear group over $p$-adic fields. J. Math. Soc. Japan 18 (1966) pp.219-235.

[16] Kempe, J.: Quantum random walks - an introductory overview, Contemporary Physics 44 (2003) pp.307-327.

[17] Kendon, V.: Decoherence in quantum walks - a review, Math. Struct. in Comp. Sci. 17 (2007) pp.1169-1220.

[18] Konno, N.: Quantum Walks. In: Lecture Notes in Mathematics: 1954 (2008) pp.309452, Springer-Verlag, Heidelberg. 
[19] Konno, N., Takei, M.: The non-uniform stationary measure for discrete-time quantum walks in one dimension, Quantum Information Comutation 15 (2015) pp.10601075 .

[20] Konno, N., Sato, I.: On the relation between quantum walks and zeta functions Quantum Information Processing 11 (2012) pp.341-349.

[21] Kotani, M., Sunada, T.: Zeta functions of finite graphs. J. Math. Sci. U. Tokyo 7 (2000) pp.7-25.

[22] Matsue, K., Ogurisu, O., Segawa, E.: A note on the spectral mapping theorem of quantum walk models, Interdisciplinary Information Sciences 23 (2017) pp.105-114.

[23] Portugal, R.: Staggered quantum walks on graphs, Physical Review A 93 (2016) 062335 .

[24] Portugal, R.: Quantum Walks and Search Algorithm, Springer (2013).

[25] Ren, P., Aleksic, T., Emms, D., Wilson, R. C., Hancock, E. R.: Quantum walks, Ihara zeta functions and cospectrality in regular graphs, Quantum Information Processing 10 (2011) pp.405-417.

[26] Stefanak, M and Skoupy, S.: Perfect state transfer by means of discrete-time quantum search algorithms, on highly symmetric graphs, Physical Review A 94 (2016) 022301.

[27] Schanz, H., Smilansky, U.: Periodic-orbit theory of Anderson localization on graphs, Physical Review Letters 14 (2000) pp.1427-1430.

[28] Serre, J. -P.: Trees, Springer-Verlag, New York (1980)

[29] Stark, H. M., Terras, A. A.: Zeta functions of finite graphs and coverings. Adv. Math. 121 (1996) pp.124-165.

[30] Sunada, T.: L-Functions in Geometry and Some Applications. In: Lecture Notes in Mathematics: 1201 (1986) pp.266-284, Springer-Verlag, New York.

[31] Sunada, T.: Fundamental Groups and Laplacians (in Japanese). Kinokuniya, Tokyo (1988)

[32] Suzuki, A.: Asymptotic velocity of a position dependent quantum walk, Quantum Information Processing 15 (2016) pp.103-119.

[33] Tanner, G.: From quantum graphs to quantum random walks, Non-Linear Dynamics and Fundamental Interactions, NATO Science Series II: Mathematics, Physics and Chemistry 213 (2006) pp.69-87. 